\title{
Charge Transport Through Open, Driven Two-Level Systems with Dissipation
}

\author{
Tobias Brandes ${ }^{1}$, Ramón Aguado ${ }^{2}$, and Gloria Platero ${ }^{2}$ \\ 1-Department of Physics, University of Manchester Institute of Science and Technology (UMIST), \\ P.O. Box 88, Manchester M60 1QD, United Kingdom and \\ 2-Departamento de Teoría de la Materia Condensada, \\ Instituto de Ciencia de Materiales de Madrid, CSIC, Cantoblanco 28049, Madrid, Spain.
}

(Dated: August 26, 2018)

\begin{abstract}
We derive a Floquet-like formalism to calculate the stationary average current through an AC driven double quantum dot in presence of dissipation. The method allows us to take into account arbitrary coupling strengths both of a time-dependent field and a bosonic environment. We numerical evaluate a truncation scheme and compare with analytical, perturbative results such as the Tien-Gordon formula.
\end{abstract}

PACS numbers: $73.23 . \mathrm{Hk}$ 73.63.Kv 03.65.Yz

\section{INTRODUCTION}

Coupled quantum systems with small effective Hilbert spaces are useful tools in order to study coherence, dissipation and the interaction properties of few-particle systems. In an electronic context, an example are coupled quantum dots $1 \cdot 2.3 .4 .5$, where strong interactions between electrons 6.7 .8 define a Coulomb blockade regime with tunnel-splitted many-body ground states separated from the remaining excited states. The ultimate limit of two states defines a two-level system for the charge degree of freedom, with electrons of a fixed spin tunneling between two quantum dots. Studying transport and dissipation then leads to a non-equilibrium or 'open' (pseudo)spin-boson problem, where the coupling to external reservoirs opens the path to investigate properties such as shot noise $e^{9}$ or decoherence in a controllable semiconductor environment.

Additional insight into the quantum dynamics of electrons can be gained by making the parameters of the problem time-dependent. When the time-dependence is slow, this can give rise to a variety of adiabatic phenomena such as charge pumping $10,11,12.13,14.15,16.17 .18,19$, adiabatic control of state vectors ${ }^{20.21}$, or operations relevant for quantum information processing in a condensedmatter setting 22.23.24.25.26.27.28. Different physics occurs in the high frequency regime where monochromatic timevariation induces photo-excitations, such as for coupling of AC fields to quantum dots29.30.31.32.33.34, which has been tested experimentally 35.36 .37 .38 .39 .40 recently.

In general, AC driven systems ${ }^{41.42}$ and their application to various mesoscopic transport 43,44,45,46,47,48,49,50

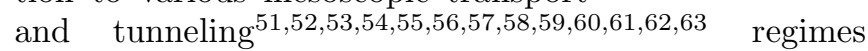
have quite a long history, although the inclusion of interactions and correlations is a relatively new area. In low-dimensional systems, investigations have concentrated on one-dimensional models ${ }^{64,65.66 .67 .68}$, the modification of Kondo-resonances by AC fields 69.70 , mean-field type approximations $\frac{71}{}$, or exact studies of driven few-electron systems 72.73 .

In this paper, we combine $\mathrm{AC}$ driving with the dissipative dynamics of a two-level system (double quantum dot) under transport conditions, i.e. in a situation where electrons in the Coulomb blockade regime can tunnel from reservoirs into and off two tunnel-coupled quantum dots, with the possibility to absorb from or emit bosons into a heat bath while simultaneously interacting with a classical time-periodic electrical field. At first sight, combining such a multitude of possible interactions within one and the same model might look unsuitable for a useful theoretical discussion. However, as we will demonstrate in this paper, it is possible to calculate experimentally relevant observables such as the time-averaged stationary current, with the help of the (heat bath) boson spectral density $J(\omega)$ as single, main input of the theory only. In particular, we show how within the polaron transformation approach and for a given $J(\omega)$, one can calculate the current for arbitrarily strong coupling to bosonic modes and an AC field.

The paper is organized as follows: in section II, we describe the model Hamiltonian and derive a Floquet-like formalism for the stationary density operator. In section III, we compare analytical results for limiting cases with numerical data, and conclude with a short discussion and an outlook in section IV.

\section{MASTER EQUATION FORMALISM FOR AC DRIVEN DOUBLE QUANTUM DOTS}

In the following, we shall develop the general framework leading to explicit expressions for the stationary current through dissipative, driven double quantum dots. Our approach is in part similar to the treatment of closed, dissipative two-level systems with $\mathrm{AC}$ driving as reviewed by Grifoni and Hänggi ${ }^{74}$. Here, we generalise this approach to take into account tunneling between the dots and the leads. In the non-dissipative case, this problem was treated by Gurvitz and Prager ${ }^{75.76}$ for non-driven double dots, and for coherently AC driven double dots by Stoof and Nazarov ${ }^{33}$. 


\section{A. Model Hamiltonian}

We assume that the driven two-level system is defined in a double quantum dot device ${ }^{3}$. In the regime of strong Coulomb blockade, these can be tuned into a regime where the internal dynamics is governed by a time-dependent (pseudo) spin-boson model (dissipative two-level system $\left.{ }^{77}\right), \mathcal{H}_{S B}(t)$. The latter describes one additional 'transport' electron which tunnels between a left $(\mathrm{L})$ and a right $(\mathrm{R})$ dot with time-dependent energy difference $\varepsilon(t)$ and inter-dot coupling $T_{c}(t)$, and is coupled to a dissipative bosonic bath $\left(\mathcal{H}_{B}=\sum_{\mathbf{Q}} \omega_{Q} a_{\mathbf{Q}}^{\dagger} a_{\mathbf{Q}}\right)$,

$$
\begin{aligned}
\mathcal{H}_{S B}(t) & =\left[\frac{\varepsilon(t)}{2}+\sum_{\mathbf{Q}} \frac{g_{Q}}{2}\left(a_{-\mathbf{Q}}+a_{\mathbf{Q}}^{\dagger}\right)\right] \hat{\sigma}_{z} \\
& +T_{c}(t) \hat{\sigma}_{x}+\mathcal{H}_{B} .
\end{aligned}
$$

The effective Hilbert space of double dot (without any coupling to electron leads or bosons) then consists of two (many-body) states $|L\rangle=\left|N_{L}+1, N_{R}\right\rangle$ and $|R\rangle=$ $\left|N_{L}, N_{R}+1\right\rangle$ and is defined by a pseudospin $\hat{\sigma}_{z} \equiv|L\rangle\langle L|-$ $|R\rangle\langle R| \equiv \hat{n}_{L}-\hat{n}_{R}$ and $\hat{\sigma}_{x} \equiv|L\rangle\langle R|+| R\rangle\langle L| \equiv \hat{p}+\hat{p}^{\dagger}$.

The effects of the bosonic bath are fully described as usual by a spectral density

$$
J(\omega) \equiv \sum_{\mathbf{Q}}\left|g_{Q}\right|^{2} \delta\left(\omega-\omega_{Q}\right),
$$

where $\omega_{Q}$ are the frequencies of the bosons and the $g_{Q}$ denote interaction constants. When showing particular results we will be using

$$
J(\omega)=2 \alpha \omega e^{-\omega / \omega_{c}},
$$

corresponding to a generic Ohmic bath. More realistic forms can be easily incorporated into our formalism but for simplicity, in this work we restrict ourselves to the Ohmic case.

The coupling to external free electron reservoirs $\mathcal{H}_{\text {res }}=\sum_{k_{\alpha}} \epsilon_{k_{\alpha}} c_{k_{\alpha}}^{\dagger} c_{k_{\alpha}}$ is described by the usual tunnel Hamiltonian

$$
\mathcal{H}_{T}=\sum_{k_{\alpha}}\left(V_{k}^{\alpha} c_{k_{\alpha}}^{\dagger} s_{\alpha}+\text { H.c. }\right)
$$

$\hat{s}_{\alpha}=|0\rangle\langle\alpha|(\alpha=\mathrm{L}, \mathrm{R})$. Here, a third state $|0\rangle=\left|N_{L}, N_{R}\right\rangle$ describes an 'empty' DQD. Its presence leads to strong modifications both in the mathematical description as well in the physics of this problem, as compared to the case of an isolated spin-boson Hamiltonian. Here, the reservoir-related parameters of $\mathcal{H}_{\text {res }}$ and $\mathcal{H}_{T}$ have been assumed to be time-independent which again is an approximation which might not be always fulfilled in experiments. Again, we concentrate on the simplest possible case in this work and neglect the effect of, e.g., a timedependence in the external electro-chemical potentials.

The full model as described by

$$
\mathcal{H}(t)=\mathcal{H}_{S B}(t)+\mathcal{H}_{\text {res }}+\mathcal{H}_{T}
$$

now offers the possibility to study non-equilibrium properties of a time-dependent, 'open' dissipative two-level system. Note that in spite of the third, 'empty' state $|0\rangle$ we continue to use the term 'two-level system' here and in the following: although the presence of $|0\rangle$ leads to strong modifications of, e.g., the equations of motion of the density operator, it turns out that the internal dynamics of the system is still closely related to that of the dissipative spin boson problem.

The time-dependent spin-boson problem is in general characterised by the fact that both $\varepsilon(t)$ and $T_{c}(t)$ are timedependent. One can then investigate interesting effects such as adiabatic charge pumping, dissipative LandauZener tunneling ${ }^{78}$, or for the closed system (no coupling to the leads) the control of quantum superpositions 79 . Although this general time-dependence offers the richest spectrum of possible physical phenomena, one is clearly strongly restricted by the fact that nearly no analytical solutions are available. In this paper, our goal is to develop a systematic theory for the stationary state of a somewhat simpler situation, i.e., the case where $T_{c}(t) \equiv T_{c}$ is constant, with the time-dependence solely contained in the bias $\varepsilon(t)$.

\section{B. Equations of Motion}

In the following, we treat the coupling to the reservoirs within the Born and Markov approximation with respect to $\mathcal{H}_{T}{ }^{33,80}$, such that higher order effects like cotunneling or the Kondo effect are not considered. This Born-Markov approximation becomes exact in the limiting case of infinite source-drain voltage ${ }^{75}$. Specifically, one sets the Fermi distributions for the left (right) reservoir $f_{L}=1\left(f_{R}=0\right)$ whence the chemical potentials of the leads no longer play any role. Furthermore, the tunnel rates which are given by

$$
\Gamma_{\alpha}=2 \pi \sum_{k_{\alpha}}\left|V_{k}^{\alpha}\right|^{2} \delta\left(\epsilon-\epsilon_{k_{\alpha}}\right), \quad \alpha=L / R,
$$

are assumed to be independent of energy. We mention that the generalisation to intermediate voltage regimes (finite bias) for double dots is a difficult and non-trivial problem even in the undriven case, which is why we only discuss the infinite-bias limit in this paper.

The derivation of the equations of motion for the dot observables is now very similar to the non-driven $\operatorname{case}^{80}$. The time-dependence of the Hamiltonian enters via the replacement of the phase factors $e^{i \varepsilon\left(t-t^{\prime}\right)}$ in the free un-driven time-evolution of the dots, by $e^{i \int_{t^{\prime}}^{t} d s \varepsilon(s)}$ for the driven case. Introducing the vectors $\mathbf{A} \equiv\left(\hat{n}_{L}, \hat{n}_{R}, \hat{p}, \hat{p}^{\dagger}\right), \mathbf{\Gamma}=\Gamma_{L} \mathbf{e}_{1}\left(\mathbf{e}_{1}, \ldots, \mathbf{e}_{4}\right.$ are unit vectors) and a time-dependent matrix memory kernel $M$, the equations of motion (EOM) can be formally written $\operatorname{as}^{9}[\langle..\rangle \equiv \operatorname{Tr} . . \rho(t)]$,

$$
\langle\mathbf{A}(t)\rangle=\langle\mathbf{A}(0)\rangle+\int_{0}^{t} d t^{\prime}\left\{M\left(t, t^{\prime}\right)\left\langle\mathbf{A}\left(t^{\prime}\right)\right\rangle+\mathbf{\Gamma}\right\} .
$$


This formulation is a useful starting point for, e.g., the calculation of shot noise. Note that in contrast to the undriven case, the memory kernel $M$ depends on both times $t$ and $t^{\prime}$ because there is no time-translation invariance in presence of driving. Explicitely, the equations for the dot expectation values read

$$
\begin{aligned}
\frac{\partial}{\partial t}\left\langle n_{L}\right\rangle_{t} & =-i T_{c}\left\{\langle p\rangle_{t}-\left\langle p^{\dagger}\right\rangle_{t}\right\}+\Gamma_{L}\left[1-\left\langle n_{L}\right\rangle_{t}-\left\langle n_{R}\right\rangle_{t}\right] \\
\frac{\partial}{\partial t}\left\langle n_{R}\right\rangle_{t} & =i T_{c}\left\{\langle p\rangle_{t}-\left\langle p^{\dagger}\right\rangle_{t}\right\}-\Gamma_{R}\left\langle n_{R}\right\rangle_{t} \\
\langle p\rangle_{t} & =-\int_{0}^{t} d t^{\prime} e^{i \int_{t^{\prime}}^{t} d s \varepsilon(s)}\left[\left(\frac{\Gamma_{R}}{2}\langle p\rangle_{t^{\prime}}+i T_{c}\left\langle n_{L}\right\rangle_{t^{\prime}}\right) C\left(t-t^{\prime}\right)-i T_{c}\left\langle n_{R}\right\rangle_{t^{\prime}} C^{*}\left(t-t^{\prime}\right)\right] \\
\left\langle p^{\dagger}\right\rangle_{t} & =-\int_{0}^{t} d t^{\prime} e^{-i \int_{t^{\prime}}^{t} d s \varepsilon(s)}\left[\left(\frac{\Gamma_{R}}{2}\left\langle p^{\dagger}\right\rangle_{t^{\prime}}-i T_{c}\left\langle n_{L}\right\rangle_{t^{\prime}}\right) C^{*}\left(t-t^{\prime}\right)+i T_{c}\left\langle n_{R}\right\rangle_{t^{\prime}} C\left(t-t^{\prime}\right)\right] .
\end{aligned}
$$

Here, half the decay rate (tunnel rate $\Gamma_{R} / 2$ ) of the system appears in the off-diagonal terms $p$ and $p^{\dagger}$, acting as a source of dephasing due to tunneling of an electron out of the dot in either direction. Furthermore, the boson correlation function for a harmonic bath with spectral density $J(\omega)$, Eq. (2) and at equilibrium temperature $k_{B} T=1 / \beta$ enters,

$$
\begin{aligned}
C(t) & \equiv e^{-Q(t)} \\
Q(t) & \equiv \int_{0}^{\infty} d \omega \frac{J(\omega)}{\omega^{2}}\left[(1-\cos \omega t) \operatorname{coth}\left(\frac{\beta \omega}{2}\right)+i \sin \omega t\right] .
\end{aligned}
$$

In deriving, the equations for the off-diagonal elements $\left\langle p^{(\dagger)}\right\rangle$, we used the polaron transformation (POL) and factorised the bosonic correlation functions from the dot operators in the equations of motions for the reduced density operator of the (pseudo) spin-boson system. This means that Eq. (8) is perturbative (though to infinite order) in the interdot coupling $T_{c}$.

Alternatively, one can perform a perturbation theory in the electron-boson coupling $g_{Q}$, (weak coupling 'PER' approach). In a calculation for an undriven double quantum dot, both approaches have been compared recently for the stationary current ${ }^{81}$ and the frequencydependent current noise $\frac{9}{-}$. For the spin-boson problem with $\Gamma_{R / L}=0$, it is well-known that POL is equivalent to a double-path integral 'non-interacting blip approximation' (NIBA) that works well for zero bias $\varepsilon=0$ but for $\varepsilon \neq 0$ does not coincide with PER at small couplings and very low temperatures. PER works in the correct bonding and anti-bonding eigenstate basis of the hybridized system, whereas the energy scale $\varepsilon$ in POL is that of the two isolated dots $\left(T_{c}=0\right)$. This difference reflects the general dilemma of two-level-boson Hamiltonians: either one is in the correct basis of the hybridized two-level system and perturbative in $g_{Q}$, or one starts from the 'shifted oscillator' polaron picture that becomes correct for $T_{c}=0$. In fact, the polaron (NIBA) approach does not coincide with standard damping theory 82 because it does not incorporate the square-root, non- perturbative in $T_{c}$ hybridization form of the level splitting $\Delta=\sqrt{\varepsilon^{2}+4 T_{c}^{2}}$. However, for large $|\varepsilon| \gg T_{c}, \Delta \rightarrow|\varepsilon|$, and POL and PER turn out to agree very well for the undriven $\operatorname{case}^{81}$.

\section{Stationary Quantities}

In a quantum system that is continuously driven by an external, time-dependent source, stationary quantities can be defined for expectation values approaching a fixed point or a quasi-stationary, periodic motion for large times $t$. In particular, we will be interested in quantities like the time-averaged electronic current. It is then useful to split the time-dependent part off $\varepsilon(t)$ as

$$
\varepsilon(t)=\varepsilon+\tilde{\varepsilon}(t)
$$

and to introduce the Laplace transform $\hat{f}(z)=$ $\int_{0}^{\infty} d t e^{-z t} f(t)$ of a function $f(t)$. The time-evolution of the isolated spin-boson system for $T_{c}=0$ is governed by the correlation function $C(t)=C^{*}(-t)$. The Laplace transform of these,

$$
\begin{aligned}
& \hat{C}_{\varepsilon}(z) \equiv \int_{0}^{\infty} d t e^{-z t} e^{i \varepsilon t} C(t) \\
& \hat{C}_{\varepsilon}^{*}(z) \equiv \int_{0}^{\infty} d t e^{-z t} e^{-i \varepsilon t} C^{*}(t) .
\end{aligned}
$$

defines free propagators for quasiparticles in the uncoupled dots and in absence of coupling to electron reservoirs. In absence of electron-boson coupling, this simply describes the free time evolution of a particle described by the diagonal Hamiltonian $\varepsilon \hat{\sigma}_{z}$, whereas for non-zero boson coupling these become 'dressed' polarons. In addition, the decay via the right reservoir at rate $\Gamma_{R}$ leads to a finite quasiparticle life-time and consequently a renor- 
malisation of the propagators as

$$
\begin{aligned}
\hat{D}_{\varepsilon}(z) \equiv \frac{\hat{C}_{\varepsilon}(z)}{1+\Gamma_{R} \hat{C}_{\varepsilon}(z) / 2}, & \hat{E}_{\varepsilon}(z) \equiv \frac{\hat{C}_{-\varepsilon}^{*}(z)}{1+\Gamma_{R} \hat{C}_{\varepsilon}(z) / 2} \\
\hat{D}_{\varepsilon}^{*}(z) \equiv \frac{\hat{C}_{\varepsilon}^{*}(z)}{1+\Gamma_{R} \hat{C}_{\varepsilon}^{*}(z) / 2}, & \hat{E}_{\varepsilon}^{*}(z) \equiv \frac{\hat{C}_{-\varepsilon}(z)}{1+\Gamma_{R} \hat{C}_{\varepsilon}^{*}(z) / 2},
\end{aligned}
$$

These expressions appear in the calculation in Appendix A, where Eq. (8) is solved for the coherences $\langle p\rangle$ and $\left\langle p^{\dagger}\right\rangle$ in order to obtain two closed equations for the occupancies $\left\langle n_{L / R}\right\rangle$,

$$
\begin{aligned}
z \hat{n}_{L}(z)-\left\langle n_{L}\right\rangle_{0} & =-\int_{0}^{\infty} d t e^{-z t}\left[\left\langle n_{L}\right\rangle_{t} \hat{K}(z, t)-\left\langle n_{R}\right\rangle_{t} \hat{G}(z, t)\right]+\Gamma_{L}\left[\frac{1}{z}-\hat{n}_{L}(z)-\hat{n}_{R}(z)\right] \\
z \hat{n}_{R}(z)-\left\langle n_{R}\right\rangle_{0} & =\int_{0}^{\infty} d t e^{-z t}\left[\left\langle n_{L}\right\rangle_{t} \hat{K}(z, t)-\left\langle n_{R}\right\rangle_{t} \hat{G}(z, t)\right]-\Gamma_{R} \hat{n}_{R}(z) \\
\hat{K}(z, t) & \equiv \int_{0}^{\infty} d t^{\prime} e^{-z t^{\prime}}\left[T_{c}\left(t+t^{\prime}\right) T_{c}^{*}(t) D_{\varepsilon}\left(t^{\prime}\right)+T_{c}^{*}\left(t+t^{\prime}\right) T_{c}(t) D_{\varepsilon}^{*}\left(t^{\prime}\right)\right] \\
\hat{G}(z, t) & \equiv \int_{0}^{\infty} d t^{\prime} e^{-z t^{\prime}}\left[T_{c}\left(t+t^{\prime}\right) T_{c}^{*}(t) E_{\varepsilon}\left(t^{\prime}\right)+T_{c}^{*}\left(t+t^{\prime}\right) T_{c}(t) E_{\varepsilon}^{*}\left(t^{\prime}\right)\right],
\end{aligned}
$$

where here and in the following we omit the $\langle.$.$\rangle in the$ Laplace transformed expectation values to simplify the notation, and we defined

$$
T_{c}(t) \equiv T_{c} e^{+i \int_{0}^{t} d s \tilde{\varepsilon}(s)}, \quad T_{c}^{*}(t) \equiv T_{c} e^{-i \int_{0}^{t} d s \tilde{\varepsilon}(s)}
$$

Up to here the transformations have been valid for an arbitrary time-dependence in $\varepsilon(t)$. ¿From now on, we specify to the time-periodic form

$$
\varepsilon(t)=\varepsilon(t+2 \pi / \Omega),
$$

where $2 \pi / \Omega$ is the period of the time-dependent field (we further specify to a sinusoidal time-dependence of $\varepsilon(t)$ below).

We expect the system to approach an asymptotic quasi-stationary state. Then, the time-evolution of all quantities $f(t)$ can be decomposed into Fourier series

$$
f(t) \rightarrow f^{a s}(t)=\sum_{n} e^{-i n \Omega t} f_{n}
$$

with multiples of the angular frequency $\Omega$ of the external field. Following Grifoni and Hänggi ${ }^{74}$, we decompose
$\hat{K}(z, t)$ and $\hat{G}(z, t)$ into Fourier series,

$$
\begin{aligned}
\hat{K}(z, t) & =\sum_{m} K_{m}(z) e^{-i m \Omega t} \\
\hat{G}(z, t) & =\sum_{m} G_{m}(z) e^{-i m \Omega t}
\end{aligned}
$$

The corresponding Fourier expansions $\left\langle n_{L}\right\rangle_{t}^{a s y} \equiv$ $\sum_{m} \nu_{m} e^{-i m \Omega t}$ and $\left\langle n_{R}\right\rangle_{t}^{a s y} \equiv \sum_{m} \mu_{m} e^{-i m \Omega t}$ of the asymptotic occupancies can then easily be Laplace transformed,

$$
\hat{n}_{L}^{a s y}(z)=\sum_{m} \frac{\nu_{m}}{z+i m \Omega}, \quad \hat{n}_{R}^{a s y}(z)=\sum_{m} \frac{\mu_{m}}{z+i m \Omega}
$$

and inserted back into Eq. (13). Comparing the complex poles at $z=-i M \Omega$ in the two equations for $\hat{n}_{L}(z)$ and $\hat{n}_{R}(z)$ and assuming that $K_{m}(z)$ and $G_{m}(z)$ are regular there, one obtains an infinite system of linear equations for the Fourier coefficients $\nu_{m}$ and $\mu_{m}$,

$$
\begin{aligned}
-i M \Omega \nu_{M} & =-\sum_{n}\left[\nu_{n} K_{M-n}(-i M \Omega)-\mu_{n} G_{M-n}(-i M \Omega)\right]+\Gamma_{L}\left[\delta_{M, 0}-\nu_{M}-\mu_{M}\right] \\
{\left[\Gamma_{R}-i M \Omega\right] \mu_{M} } & =\sum_{n}\left[\nu_{n} K_{M-n}(-i M \Omega)-\mu_{n} G_{M-n}(-i M \Omega)\right] .
\end{aligned}
$$

Upon adding these two equations, one has

$$
-\frac{\mu_{M}}{\nu_{M}} \equiv r_{M} \equiv\left[1+\frac{\Gamma_{R}}{\Gamma_{L}-i M \Omega}\right]^{-1}, \quad M \neq 0,
$$

and Eq. 19) can be transformed into a single matrix equation for the coefficients $\nu_{n}$,

$$
\sum_{n=-\infty}^{\infty} A_{m n} \nu_{n}=b_{m}
$$


where

$$
\begin{aligned}
A_{m n} & \equiv\left(\Gamma_{L}-i n \Omega-r_{n} \Gamma_{L}\right) \delta_{m, n} \\
& +K_{m-n}(-i m \Omega)+r_{n} G_{m-n}(-i m \Omega) \\
b_{m} & \equiv \frac{\Gamma_{L} \Gamma_{R}}{\Gamma_{R}+\Gamma_{L}} \delta_{m 0}+\frac{\Gamma_{L}}{\Gamma_{R}+\Gamma_{L}} G_{m}(-i m \Omega) .
\end{aligned}
$$

\section{Charge Current}

In the Master equation approach, the expectation values of the electron current through the double dot is obtained in a fairly easy manner. One has to consider the average charge flowing through one of the three intersections, i.e., left lead/left dot, left dot/right dot, and right dot/right lead. This gives rise to the three corresponding electron currents $I_{L}(t), I_{R}(t)$, and the interdot current $I_{L R}(t)$. ¿From the equations of motion, Eq. (8), one recognises that the temporal change of the occupancies $\left\langle n_{L / R}\right\rangle_{t}$ is due to the sum of an 'interdot' current $\propto T_{c}$ and a 'lead-tunneling' part. Specifically, the current from left to right through the left (right) tunnel barrier is

$$
\begin{aligned}
& I_{L}(t)=-e \Gamma_{L}\left\langle n_{0}\right\rangle_{t}=-e \Gamma_{L}\left[1-\left\langle n_{L}\right\rangle_{t}-\left\langle n_{R}\right\rangle_{t}\right] \\
& I_{R}(t)=-e \Gamma_{R}\left\langle n_{R}\right\rangle_{t},
\end{aligned}
$$

and the interdot current is

$$
\begin{aligned}
I_{L R}(t) & =-i e T_{c}\left\{\langle p\rangle_{t}-\left\langle p^{\dagger}\right\rangle_{t}\right\} \\
& =-e \frac{\partial}{\partial t}\left\langle n_{R}\right\rangle_{t}+I_{R}(t)=e \frac{\partial}{\partial t}\left\langle n_{L}\right\rangle_{t}+I_{L}(t) .
\end{aligned}
$$

In the stationary case, all the three currents are the same: adding the two equations Eq. (19) for $M=0$, we first obtain

$$
\Gamma_{R} \mu_{0}=\Gamma_{L}\left(1-\mu_{0}-\nu_{0}\right) .
$$

Using furthermore the Fourier expansion of $\left\langle n_{L / R}\right\rangle_{t}^{a s y}$, we recognize from Eq. (2324) that

$$
\bar{I}=\overline{I_{L}(t)}=\overline{I_{L R}(t)}=\overline{I_{R}(t)}=-e \Gamma_{R} \mu_{0},
$$

where the bar denotes the temporal average of the asymptotic quantities over one period $\tau \equiv 2 \pi / \Omega$. This simple result means that the stationary current is determined by the Fourier component $\mu_{0}$ only. Note, however, that $\mu_{0}$ is part of the solution of an infinite set of the linear equations Eq. (21). Using Eq. (19) and Eq. (20), one can express $\mu_{0}$ in terms of $\mu_{N \neq 0}$ for the alternative expression

$$
\bar{I}=-e \Gamma_{R} \frac{K_{0}(0)-\sum_{n \neq 0}\left[K_{-n}(0) / r_{n}+G_{-n}(0)\right] \mu_{n}}{\Gamma_{R}+K_{0}(0) / r_{0}+G_{0}(0)}
$$

This form is in particular useful for the discussion of the Tien-Gordon limit below.

\section{E. Sinusoidal Time-Dependence}

In the following, we specify the time-dependence of the bias $\varepsilon(t)$ to a monochromatic sinusoidal field

$$
\varepsilon(t)=\varepsilon+\Delta \sin (\Omega t)
$$

where the constant part is denoted by $\varepsilon$. We introduce the notation,

$$
\begin{aligned}
& \hat{C}_{\varepsilon}(-i \omega)=\hat{C}_{\varepsilon+\omega}\left(z=0^{+}\right) \equiv \hat{C}_{\varepsilon+\omega} \\
& \hat{C}_{\varepsilon}^{*}(-i \omega)=\hat{C}_{\varepsilon-\omega}^{*}\left(z=0^{+}\right) \equiv \hat{C}_{\varepsilon-\omega}^{*}
\end{aligned}
$$

and correspondingly for the propagators $D, D^{*}, E$, and $E^{*}$, Eq. (12). Then, invoking the decomposition of the phase factor into Bessel functions,

$$
\begin{aligned}
& T_{c}\left(t+t^{\prime}\right) T_{c}^{*}\left(t^{\prime}\right)=T_{c}^{2} e^{i \int_{t}^{t+t^{\prime}} d s \Delta \sin (\Omega s)}= \\
& T_{c}^{2} \sum_{n n^{\prime}} i^{n^{\prime}-n} J_{n}\left(\frac{\Delta}{\Omega}\right) J_{n^{\prime}}\left(\frac{\Delta}{\Omega}\right) e^{-i n \Omega t^{\prime}} e^{-i\left(n-n^{\prime}\right) \Omega t}
\end{aligned}
$$

and the definitions of the Fourier components $K_{m}(z)$ and $G_{m}(z)$, cf. Eq. (17) and Eq. (13), one obtains

$$
\begin{aligned}
& K_{m}\left(-i m^{\prime} \Omega\right)=i^{-m} T_{c}^{2} \sum_{n}\left[J_{n}\left(\frac{\Delta}{\Omega}\right) J_{n-m}\left(\frac{\Delta}{\Omega}\right) \hat{D}_{\varepsilon+\left(m^{\prime}-n\right) \Omega}+J_{n}\left(\frac{\Delta}{\Omega}\right) J_{n+m}\left(\frac{\Delta}{\Omega}\right) \hat{D}_{\varepsilon-\left(m^{\prime}+n\right) \Omega}^{*}\right] \\
& G_{m}\left(-i m^{\prime} \Omega\right)=i^{-m} T_{c}^{2} \sum_{n}\left[J_{n}\left(\frac{\Delta}{\Omega}\right) J_{n-m}\left(\frac{\Delta}{\Omega}\right) \hat{E}_{\varepsilon+\left(m^{\prime}-n\right) \Omega}+J_{n}\left(\frac{\Delta}{\Omega}\right) J_{n+m}\left(\frac{\Delta}{\Omega}\right) \hat{E}_{\varepsilon-\left(m^{\prime}+n\right) \Omega}^{*}\right] .
\end{aligned}
$$

\section{ANALYTICAL RESULTS}

In the following, we first discuss the limits where analytical results for the stationary current $\bar{I}$ can be ob- tained, and then turn to a comparison with numerical calculations. 


\section{A. Time-Independent Case}

For $\Delta=0$, i.e. in absence of the time-dependent (driving) part in $\varepsilon(t)$, we recover previous results 80 for stationary transport in dissipative double quantum dots. One then has $\hat{K}(z, t)=\hat{K}(z)$ and $\hat{G}(z, t)=\hat{G}(z)$ such that $K_{n}(z)=G_{n}(z)=0$ for $n \neq 0$. Using $K_{0}(0)=2 \operatorname{Re}\left[T_{c}^{2} \hat{C}_{\varepsilon} /\left(1+\Gamma_{R} \hat{C}_{\varepsilon} / 2\right)\right]$, together with $G_{0}(0)=2 \operatorname{Re} T_{c}^{2}\left[\hat{C}_{-\varepsilon}^{*} /\left(1+\Gamma_{R} \hat{C}_{\varepsilon} / 2\right)\right]$, after some algebra we re-derive the previous result ${ }^{80}$ for the stationary current,

$$
\begin{gathered}
\bar{I}=-e T_{c}^{2} \frac{2 \operatorname{Re}\left(\hat{C}_{\varepsilon}\right)+\Gamma_{R}\left|\hat{C}_{\varepsilon}\right|^{2}}{\left|1+\Gamma_{R} \hat{C}_{\varepsilon} / 2\right|^{2}+2 T_{c}^{2} B_{\varepsilon}} \\
B_{\varepsilon} \equiv \operatorname{Re}\left\{\left(1+\Gamma_{R} \hat{C}_{\varepsilon} / 2\right)\left[\frac{\hat{C}_{-\varepsilon}}{\Gamma_{R}}+\frac{\hat{C}_{\varepsilon}^{*}}{\Gamma_{L}}\left(1+\frac{\Gamma_{L}}{\Gamma_{R}}\right)\right]\right\}
\end{gathered}
$$

(note the absence of the factor 2 in the definition of the rates here $\left.{ }^{80}\right)$. The result Eq. (32), which can be compared 81 to an alternative derivation using perturbation theory in the boson coupling $\alpha$, generalizes the case of elastic tunneling through double quantum dots to inelastic tunneling with coupling to an arbitrary bosonic heat bath. For $\alpha=0$, we re-derive the Stoof-Nazarov expression for the stationary current without dissipation,

$$
\bar{I}_{\alpha=0}=-e \frac{T_{c}^{2} \Gamma_{R}}{\varepsilon^{2}+\Gamma_{R}^{2} / 4+T_{c}^{2}\left(2+\Gamma_{R} / \Gamma_{L}\right)} .
$$

\section{B. Lowest order $T_{c}^{2}$ : Tien-Gordon Result}

In the time-dependent case, we are able to derive analytical results by considering the limit of small interdot coupling $T_{c}$, or large frequencies $\Omega$. These two limits do not yield identical results because apart from $T_{c}$ and $\Omega$, there are four other energy scales (bias $\varepsilon$, rates $\Gamma_{L}, \Gamma_{L}$, boson cut-off $\omega_{c}$ ) in the problem.

Considering Eq. (33) for the undriven, non-dissipative current, lowest order perturbation theory in $T_{c}$ is valid for $T_{c} \sqrt{2+\Gamma_{R} / \Gamma_{L}} \ll \Gamma_{R},|\varepsilon|$. The additional energy scale $\Omega$ due to AC-driving requires that this condition is generalised to

$$
T_{c} \sqrt{2+\frac{\Gamma_{R}}{\Gamma_{L}}} \ll \Omega, \Gamma_{R},|\varepsilon+n \Omega|, \quad n= \pm 0,1,2, . .,
$$

which indicates that at the resonance points $\varepsilon=n \Omega$ such a perturbation theory must break down, as is corroborated by our numerical results discussed below.

Considering the expression for $\mu_{M}$ in Eq. (19), one recognises that $\mu_{M}=O\left(T_{c}^{2}\right)$ because the Fourier components of the functions $K$ and $G$ are proportional to $T_{c}^{2}$, cf. Eq. (13). Owing to the full expression Eq. (27), the stationary current in lowest order of $T_{c}$ is $\bar{I}=\bar{I}^{\mathrm{TG}}+O\left(T_{c}^{4}\right)$ with

$$
\bar{I}^{\mathrm{TG}} \equiv-e K_{0}(0)
$$

For a sinusoidal $\varepsilon(t)=\varepsilon+\Delta \sin (\Omega t)$, the explicit expression Eq. (31) yields

$$
\bar{I}^{\mathrm{TG}}=-e T_{c}^{2} \sum_{n} J_{n}^{2}\left(\frac{\Delta}{\Omega}\right) \operatorname{Re}\left(\frac{2 C_{\varepsilon+n \Omega}}{1+\frac{\Gamma_{R}}{2} C_{\varepsilon+n \Omega}}\right)
$$

Note that Eq. (36) is the Tien-Gordon formula. This can be easily demonstrated by expanding the non-driven stationary current, Eq. (32), to lowest order in $T_{c}$, namely $\bar{I}=\bar{I}_{0}+O\left(T_{c}^{4}\right)$, such that, for the driven case:

$$
\left.\bar{I}^{\mathrm{TG}} \equiv \sum_{n} J_{n}^{2}\left(\frac{\Delta}{\Omega}\right) \bar{I}_{0}\right|_{\varepsilon \rightarrow \varepsilon+n \Omega} ^{\Delta=0} .
$$

To lowest order in $T_{c}$, the stationary current therefore is given by the Tien-Gordon formula: the current in the driven system is expressed by a sum over current contributions from side-bands $\varepsilon+n \Omega$, weighted with squares of Bessel functions. Note that the perturbative result $\bar{I}^{\mathrm{TG}} \equiv-e K_{0}(0)$, Eq. (35), does not refer to any specific form of the periodic function $\varepsilon(t)$; it is valid for arbitrary periodic driving when the corresponding Fourier component $K_{0}(0)$ is used.

\section{Non-Adiabatic Approximation}

This approximation assumes that the frequency $\Omega$ is the largest energy scale in the problem,

$$
\Omega \gg T_{c}, \varepsilon, \Gamma_{R}, \Gamma_{L} .
$$

On the r.h.s. of the integral equation Eq. (13) for $\hat{n}_{L / R}(z)$, one then replaces the integral kernels $\hat{K}(z, t)$ and $\hat{G}(z, t)$ by their averages over one period of the AC field,

$$
\hat{K}(z, t) \rightarrow \frac{\Omega}{2 \pi} \int_{0}^{2 \pi / \Omega} d t \hat{K}(z, t) \equiv K_{0}(z)
$$

and similarily for $\hat{G}(z, t)$. The Fourier coefficients $K_{n}(z)$ and $G_{n}(z)$ with $n \neq 0$ then vanish and one obtains $\bar{I} \approx$ $\bar{I}^{\text {fast }}$, where

$$
\bar{I}^{\text {fast }} \equiv \frac{-e \Gamma_{R} K_{0}(0)}{\Gamma_{R}+G_{0}(0)+K_{0}(0)\left[1+\Gamma_{R} / \Gamma_{L}\right]} .
$$

We observe that within lowest order of the static tunneling $T_{c}$, Eq. (40) coincides with the Tien-Gordon expression, Eq. (35), which one obtains by setting $G_{0}(0) \propto T_{c}^{2}$ and $K_{0}(0) \propto T_{c}^{2}$ to zero in the denominator of Eq. (40). In fact, for the undriven case $\Delta=0$ one can prove $e^{81}$ that the expression for the stationary current sums up an infinite number of terms $\propto T_{c}^{2}$, a fact that can be traced back to the integral equation structure of the underlying master equation. Here, Eq. (40) demonstrates that a similar summation effectively can be achieved in the AC driven case. 


\section{Higher Order Corrections to Tien-Gordon}

In order to systematically go beyond the Tien-Gordon approximation, Eq. (35), one has to perform an expansion of the current in powers of $T_{c}^{2}$. This can be achieved by truncating the infinite set of linear equations, Eq. (22), in order to obtain approximations for the $n=0, \pm 1, \pm 2$ th side-band values of $\nu_{n}$ and $\mu_{n}$. The simplest way to do this in practice is by a numerical solution of these equations as discussed below.

Barata and Wreszinski ${ }^{83}$ have considered higher order corrections to dynamical localisation in a closed, coherent two-level system, i.e., without coupling to external electron reservoirs or dissipation. They found that the next order in perturbation theory given a contribution different from zero was the third order one, giving a contribution to a renormalisation of the tunnel coupling $T_{c}$ :

$$
\begin{aligned}
\delta T_{c}^{(3)} \equiv-\frac{2 T_{c}^{3}}{\Omega^{2}} \times \\
\sum_{n_{1}, n_{2} \in Z} \frac{J_{2 n_{1}+1}\left(\frac{\Delta}{\Omega}\right) J_{2 n_{2}+1}\left(\frac{\Delta}{\Omega}\right) J_{-2\left(n_{1}+n_{2}+1\right)}\left(\frac{\Delta}{\Omega}\right)}{\left(2 n_{1}+1\right)\left(2 n_{2}+1\right)} .
\end{aligned}
$$

We now recall our expression

$$
K_{0}(0)=\sum_{n}\left[T_{c} J_{n}\left(\frac{\Delta}{\Omega}\right)\right]^{2} 2 \operatorname{Re} D_{\varepsilon+n \Omega}
$$

(and $G_{0}(0)$ correspondingly with $D_{\varepsilon+n \Omega}$ replaced by $\left.E_{\varepsilon+n \Omega}\right)$, cf. Eq. (31), which enter the Tien-Gordon result, Eq. (35), and the re-summed non-adiabatic approximation Eq. (40). We use the renormalised $T_{c}$, Eq. (41), in order to define a renormalised function $K_{0}^{(3)}(0)$,

$$
K_{0}^{(3)}(0) \equiv \sum_{n}\left[T_{c} J_{n}\left(\frac{\Delta}{\Omega}\right)+\delta T_{c}^{(3)}\right]^{2} 2 \operatorname{Re} D_{\varepsilon+n \Omega},
$$

and $G_{0}^{(3)}(0)$ correspondingly. This yields an expression for the current, renormalised up to third order in $T_{c}$, according to

$$
\bar{I}^{(3)} \equiv \frac{-e \Gamma_{R} K_{0}^{(3)}(0)}{\Gamma_{R}+G_{0}^{(3)}(0)+K_{0}^{(3)}(0)\left[1+\Gamma_{R} / \Gamma_{L}\right]} .
$$

In the following, we discuss and compare our above results.

\section{DISCUSSION}

\section{A. Comparison of Two Numerical Schemes}

In order to numerically solve the integro-differential system Eq. (8), it is convenient to write

$$
\exp \left(i \int_{t^{\prime}}^{t} d s \varepsilon(s)\right) \equiv e^{i \varphi_{t}} e^{-i \varphi_{t^{\prime}}}
$$

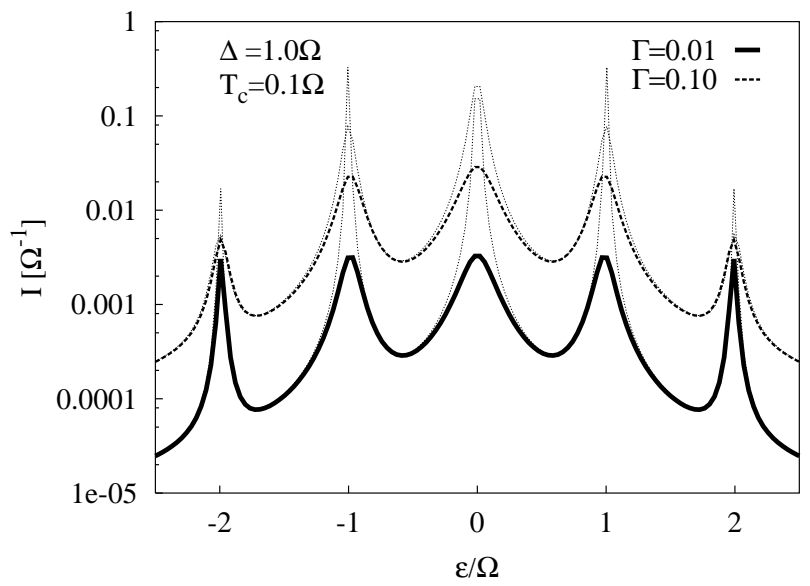

FIG. 1: Average current through double dot in Coulomb blockade regime with bias $\varepsilon+\Delta \sin \Omega t$. Coupling to left and right leads $\Gamma_{L}=\Gamma_{R}=\Gamma$. Dotted lines indicate Tien-Gordon result, Eq. (37)

with $\varphi_{t} \equiv \varepsilon t-(\Delta / \Omega) \cos \Omega t$, remembering our choice $\varepsilon(t)=\varepsilon+\Delta \sin \Omega t$. We then introduce the real and imaginary part of $\langle p\rangle$, use $e^{i x}=\cos x+i \sin x$, and specify to the Ohmic dissipation case for $C(t)$,

$$
\begin{aligned}
C(t) & =|C(t)| e^{-i \Psi_{t}}, \quad \Psi_{t}=2 \alpha \arctan \omega_{c} t \\
|C(t)| & =\left[1+\left(\omega_{c} t\right)^{2}\right]^{-\alpha}\left|\frac{\Gamma\left(1+1 / \beta \omega_{c}+i t / \beta\right)}{\Gamma\left(1+1 / \beta \omega_{c}\right)}\right|^{4 \alpha} .
\end{aligned}
$$

We have solved Eqs. (8) numerically as a function of time, with the result for large times used to obtain the stationary current as a function of $\varepsilon$. For each value of $\varepsilon$, the time-dependent equations have been solved up to a fixed final time $t_{f}$ with a subsequent time-average over the interval $\left[t_{f}-\Delta t, t_{f}\right] . t_{f}$ has to be chosen sufficiently large, in particular for larger values of $\alpha$. Consequently, one then also has to increase the number of steps to achieve sufficient accuracy of the data. We have used these numerical results to check our method for the stationary quantities as obtained from truncating Eq. (22) at a finite photo-sideband number, and found good agreement between both methods. Whereas the direct integration of the equations of motion is somewhat slower than the truncation method, it has the advantage that it does not require analytic forms of the Laplace transform for the bosonic correlation functions $\hat{C}_{\varepsilon}$, Eqs. (11) and Eqs. (29). The latter are required for the matrix scheme Eq. (22). In Appendix B we derive explicit expressions for zero temperature $(T=0)$ and Ohmic dissipation. Note

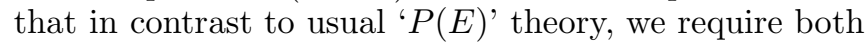
the real part $\operatorname{Re}\left[\hat{C}_{\varepsilon}(0)\right]=\pi P(\varepsilon)$ (where $P(\varepsilon)$ is the probability for inelastic tunneling with energy transfer $\varepsilon^{82}$ ), and the imaginary part of $\hat{C}_{\varepsilon}$.

In the following, we show numerical results obtained with the truncation method. 


\section{B. Photo-Sidebands (Coherent Case)}

\section{Comparison with Tien-Gordon approximation}

In Fig. (11), we compare the exact numerical result for the average stationary current with the Tien-Gordon expression, Eq. (37), in the coherent case $\alpha=0$. One clearly recognises the symmetric photo-side peaks which, according to Eq. (37), appear at $\pm n \hbar \varepsilon$. The Tien-Gordon approximation overestimates the current close to these resonances, where terms of higher order in $T_{c}$ become important due to the non-linearity (in $T_{c}$ ) of the exact bonding and antibonding energies $\pm \sqrt{\varepsilon^{2}+4 T_{c}^{2}}$ of the isolated two-level system. This again confirms that the Tien-Gordon result is perturbative in the tunneling $T_{c}$.

\section{RWA and Bloch-Siegert shift}

Close to the first side-peak, Stoof and Nazarov have used a Rotation Wave Approximation (RWA) to obtain analytical predictions for the first current side-peak. In this approximation, one transforms into an interaction picture where the fast-rotating terms with angular frequency $\pm \Omega$ are transformed away, and terms with higher rotation frequencies (such as $\pm 2 \Omega$ ) are neglected. The resulting expression for the current is ${ }^{33}$

$$
I_{\mathrm{SN}}=\frac{\Delta^{2} \Gamma_{R}\left(a^{2}-4\right)}{c\left(c \Gamma_{R}^{2}+b \Delta^{2}\right)} \frac{w^{2}}{w^{2}+\left(\varepsilon-\varepsilon_{r}\right)^{2}},
$$

with the resonance point $\varepsilon_{R} \equiv \sqrt{\Omega^{2}-4 T_{c}^{2}}$ and parameters $a=\Omega / T_{c}, b \equiv \Gamma_{R} / \Gamma_{L}+2, c \equiv a^{2}+b-4$, and the half-width $w=\left(a /\left[2 \sqrt{a^{2}-4}\right]\right) \sqrt{\Gamma_{R}^{2}+(b / c) \Delta^{2}}$. We compare $I_{\mathrm{SN}}$ with the exact result in Fig.(2).

For smaller driving amplitude $\Delta$, the agreement is very good but becomes worse with increasing $\Delta$. The position of the side-peak resonance point, which is independent of $\Delta$ in the Stoof-Nazarov approximation Eq. (47), starts to shift towards slightly larger values of the bias $\varepsilon$. In fact, for stronger AC driving the RWA is known to break down: in isolated two-level systems, the first corrections to the RWA lead to the well-known Bloch-Siegert shift ${ }^{84}$ of the central resonance towards larger energies, which is consistent with the exact result in Fig. (2).

\section{Dynamical Localization and its Lifting}

In a quantum system driven by a periodic electric field, a phenomenon termed Coherent Destruction of Tunneling (CDT) (also denoted Dynamical Localization (DL)) occurs under certain parameters of the external field ${ }^{52.85}$. The periodicity of the external field allows to write the solutions of the Schrödinger equation as: $\psi(t)=\exp \left[-i \epsilon_{j} t\right] \phi_{j}(t)$ where $\epsilon_{j}$ is called the quasienergy, and $\phi_{j}(t)$ is a function with the same period as the driving field: the Floquet state.

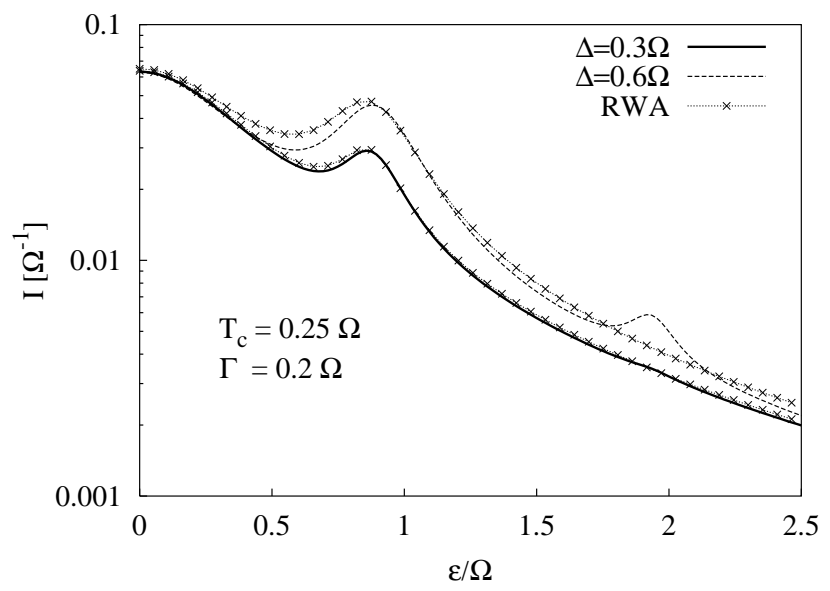

FIG. 2: Comparison between RWA, Eq. (47), and exact result for first current side-peak.

When two quasi-energies approach degeneracy, the time-scale for tunneling between the states diverges, producing the phenomenon of coherent destruction of tunneling (CDT) $)^{52}$. The time scale for localization is the inverse of the energy separation of the quasienergies.

In the case of an isolated two level system driven by a monochromatic, sinusoidal field $\varepsilon(t)=\varepsilon+\Delta \sin (\Omega t)$, Eq.(28), CDT can be physically understood from the renormalization of the coupling $T_{c}$ of the two levels,

$$
T_{c} \rightarrow T_{c, \mathrm{eff}} \equiv T_{c} J_{0}\left(\frac{\Delta}{\hbar \Omega}\right) .
$$

This expression is obtained from first-order perturbation theory in the tunneling $T_{c}{ }_{c}^{85}$. At the first zero of the Bessel function $J_{0}$, namely when $\Delta / \hbar \Omega=2.4048 \ldots$, the effective tunnel splitting vanishes, leading to a complete localization of the particle in the initial state.

In the following, we discuss how stronger tunnel amplitudes $T_{c}$, the coupling to the external leads, and dissipation modify this picture.

\section{Current Suppression}

In Fig. (3), we show results for the average current and $\alpha=0$ (no dissipation) in the dynamical localisation (DL) regime. Here, we define this regime by $\Delta=z_{0} \Omega$, where $z_{0}=2.4048 \ldots$ is the first zero of the Bessel function $J_{0}$. For this specific value of the AC driving $\Delta$, to lowest order in $T_{c}$ the average current is strongly suppressed for $|\varepsilon| \lesssim \Omega$ as compared with the un-driven case $\Delta=0$. For small $T_{c}$, this suppression is well-described by the Tien-Gordon expression (not shown here): since at $\Delta=z_{0} \Omega$, the $n=0$ term in the sum Eq. (37) is absent, the current is dominated by the shifted (un-driven) current contributions at bias $\varepsilon+n \Omega$ with $|n| \geq 1$, which however are very small due to the 

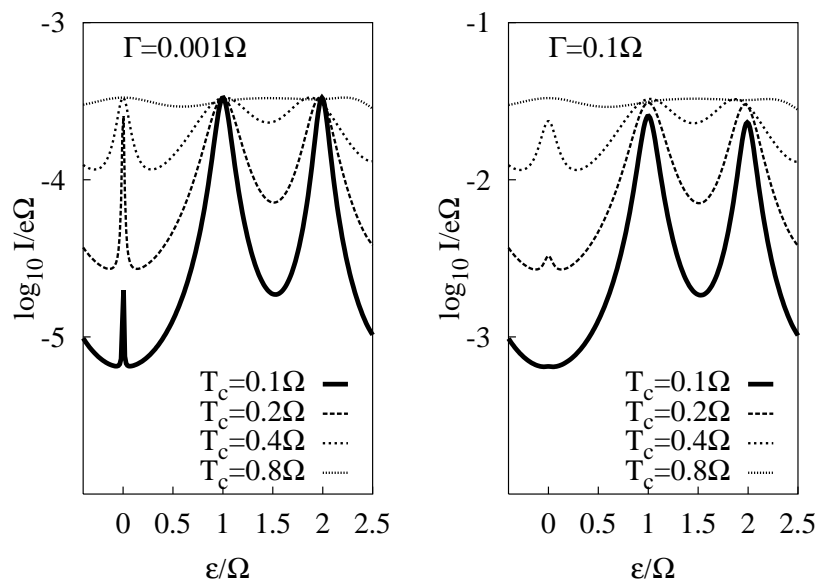

FIG. 3: Average current for AC driving amplitude $\Delta=$ $z_{0} \Omega\left(z_{0}\right.$ first zero of Bessel function $\left.J_{0}\right)$ and various tunnel couplings $T_{c}$. Coupling to left and right leads $\Gamma_{L}=\Gamma_{R}=\Gamma$.

resonance shape of the un-driven current.

\section{Central Current Peak and Third-Order Result}

Surprisingly, however, the coherent suppression of the current is lifted again very close to $\varepsilon=0$, where a small and sharp peak appears. This peak becomes broader with increasing tunnel coupling $T_{c}$, but its height is suppressed for increasing reservoir coupling $\Gamma$, cf. Fig. (3) right. This feature is analysed in Fig. (4), where we show results for the central current peak around $\varepsilon=0$ in the DL regime for coherent $(\alpha=0$, left $)$ and incoherent $(\alpha>0$, right) tunneling. As one recognises, the Tien-Gordon description (which is perturbative in the tunnel coupling $T_{c}$ ) breaks down close to $\varepsilon=0$ where higher order terms in $T_{c}$ become important. As a matter of fact, for $\varepsilon=0$ the only relevant energy scale of the isolated two-level systems is $T_{c}$ itself. In contrast, the third order approximation Eq. (44) reproduces very well the additional peak at $\varepsilon=0$, which indicates the importance of higher order terms in that regime. At $\varepsilon=0$, the charge between the two dots is strongly de-localised in the undriven case, and this tunneling-induced quantum coherence persists into the strongly driven regime where its signature is a 'lifting' of the DL close to $\varepsilon=0$.

The width of the corresponding current peak is determined by the tunneling rate $\Gamma$. An increase of incoherent electron tunneling from the left lead therefore washes out the coherent lifting of the DL. This argument in emphasised in the right part of Fig. (4) which shows that the central peak in the DL regime vanishes for increasing dissipation strength $\alpha$.
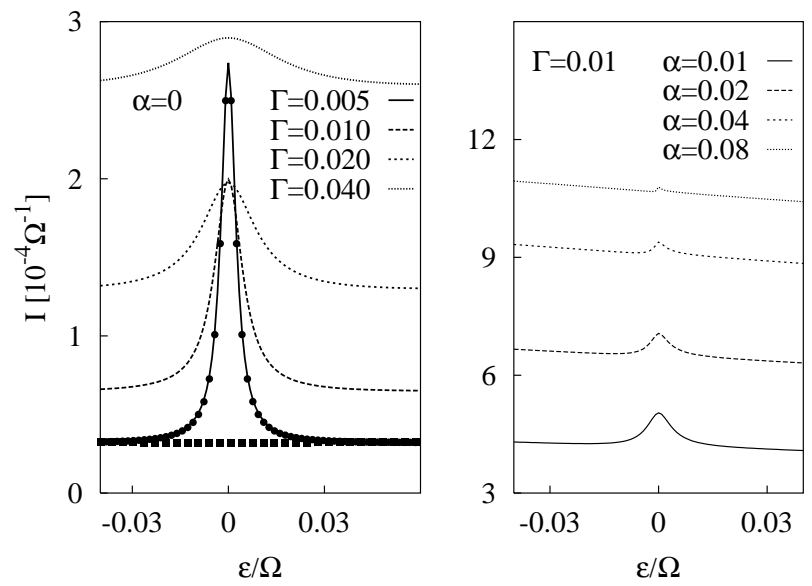

FIG. 4: Central peak of average current through AC driven double quantum dot. Parameters $T_{c}=0.1, \Delta=z_{0} \Omega$ (all rates in units of $\Omega$ ) LEFT: coherent case $\alpha=0$ for different tunnel rates $\Gamma=\Gamma_{L}=\Gamma_{R}$, dots indicate third order results Eq. (44), squares indicate the Tien-Gordon result Eq. (37) for the case $\Gamma=0.005$. RIGHT: disappearance of central peak with increasing dissipation $\alpha$.

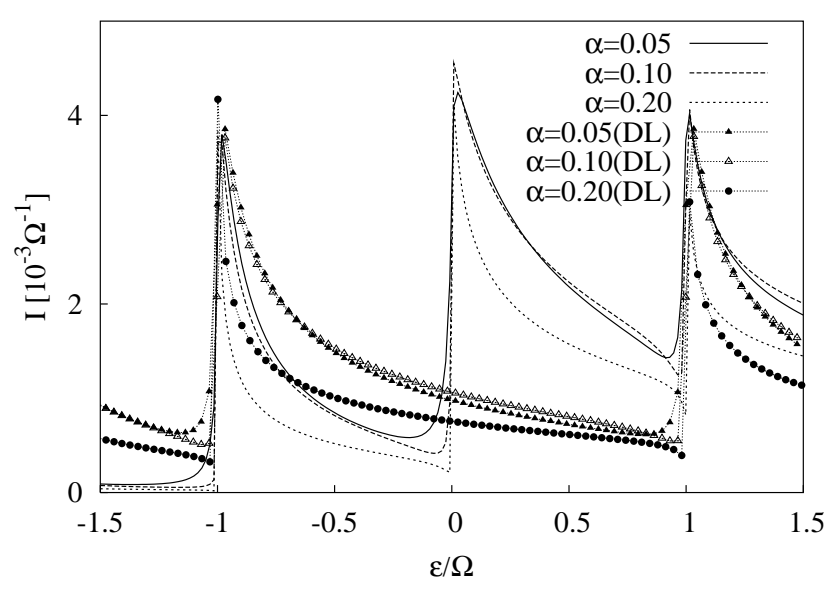

FIG. 5: Average current through double dot in Coulomb blockade regime with bias $\varepsilon+\Delta \sin \Omega t$ for various Ohmic dissipation strengths $\alpha$ at zero temperature. Driving amplitude $\Delta=\Omega$ for lines without symbols, $\Delta=z_{0} \Omega\left(z_{0}\right.$ first zero of Bessel function $J_{0}$ ) for lines with symbols. Tunnel coupling between dots $T_{c}=0.1 \Omega$, bath cutoff $\omega_{c}=500 \Omega$, and lead tunnel rates $\Gamma_{L}=\Gamma_{R}=0.01 \Omega$.

\section{Dissipation and Average Current}

\section{Dissipative Photo-Sidebands}

As mentioned above, for simplicity we restrict ourselves to an Ohmic dissipative bath at zero temperature $(T=0)$ in this paper, leaving the finite temperature case or the case of more complicated spectral functions $J(\omega)$ for future work.

For $\Delta=0$, we reproduce the analytical result Eq. (32) 


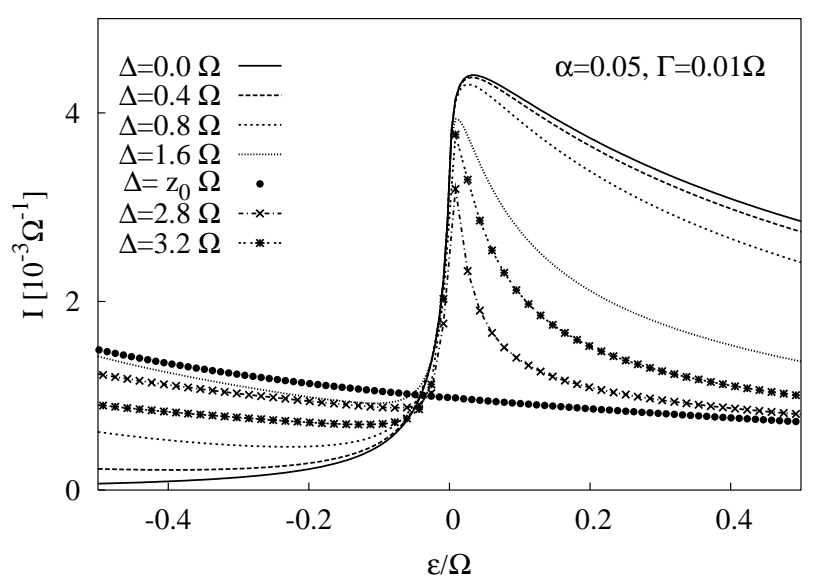

FIG. 6: Average current through driven double dot for various AC driving amplitudes $\Delta$ and fixed dissipation $\alpha=0.05$, tunnel coupling $T_{c}=0.1 \Omega$.

and the corresponding inelastic current part for $\varepsilon>0$ due to spontaneous boson emission ${ }^{3,80}$. In Fig. (15), we show the stationary current as a function of bias $\varepsilon$ for various Ohmic dissipation strengths $\alpha$ at zero temperature and finite $\mathrm{AC}$ driving amplitudes $\Delta$. For $\Delta=\Omega$, apart from the central resonant tunneling peak, side-bands at $\varepsilon=$ $n \Omega$ appear which reproduce the asymmetry of the central peak around $\varepsilon=0$. This asymmetry is a clear signature of the coupling to the dissipative environment strongly modifying the current even at zero temperature.

The specific form of the inelastic current depends on the boson spectral density $J(\omega)^{80}$. Note that in general, there is no monotonic dependence on the dissipation strength $\alpha$ since the boson correlation function $\hat{C}_{\varepsilon}$ appears both in the denominator and the numerator of the expression for the current Eq. (32).

\section{Dissipation and Dynamical Localisation in the Current}

If the $\mathrm{AC}$ driving amplitude $\Delta$ is increased towards $z_{0} \Omega\left(z_{0}\right.$ is the first zero of the Bessel function $\left.J_{0}\right)$, one expects to enter the regime of dynamical localization and a strong suppression of the central current peak. In the coherent case $\alpha=0$ (see above), resonant tunneling is usually strongly inhibited due to coherent suppression of tunneling.

For $\alpha>0$, however, we find that the current suppression strongly depends on the static bias $\varepsilon$ : we find suppression for $\varepsilon>0$ and, in general, larger values of the current for $\varepsilon<0$ as compared to the case of smaller AC amplitudes $\Delta$. We explain this feature in the following: the dependence of the average current on the driving amplitude $\Delta$ for fixed $\alpha$ is clearly visible in Fig. (6). A small driving amplitude $\Delta \lesssim 0.2$ nearly does not change the current at all. However, the originally strongly asymmetric current curve becomes flattened out when $\Delta$ is tuned to larger values up to the dynamical localization value $\Delta=z_{0} \Omega$. There, the $\mathrm{AC}$ field nearly completely destroys the strong asymmetry between the spontaneous emission $(\varepsilon>0)$ and the absorption side $(\varepsilon<0)$ of the current. The central $n=0$ photo-band is completely suppressed and the dominant contribution to the current comes from the $n= \pm 1$ bands. For $\varepsilon<0$, the current for $\Omega>|\varepsilon|$ is due to photo-excitation of the electron into the first upper photo-sidebands and subsequent spontaneous emission of bosons of energy $E_{1} \equiv \Omega-|\varepsilon|$ to the bath. In contrast, for $\Omega>\varepsilon>0$, photon emission blocks the current because at $T=0$ there is no absorption of bosons from the bath. The remaining photon absorption channel then leads to boson emission at an energy $E_{2} \equiv \Omega+\varepsilon$, which is larger as compared to the case for $\varepsilon<0$, namely $E_{2}>E_{1}$, and therefore has a smaller probability $P(E) \propto E^{2 \alpha-1} e^{-E / \omega_{c}}$, cf. Eq.(B9), leading to a smaller current. A similar argument can be used to explain why the current increases as one reduces $\varepsilon$, say from $\varepsilon / \Omega=0$ to $\varepsilon / \Omega=-0.5$. In particular, the strongest effect of the dissipative bath occur near one-photon resonance conditions, i.e. when $\varepsilon / \Omega \approx \pm 1$, where the current is regulated by the spectral function of the bosonic bath at very low frequencies, either by absorption $(\varepsilon<0)$ or emission $(\varepsilon>0)$ of a photon. These processes appear in the current as nonanalytic cusps reflecting the power law behavior of $P(E)$. This has to be compared with the Lorentzian shape of the photo-sidebands in the absence of dissipation (Fig. 1). If one tunes to even larger values of $\Delta>z_{0} \Omega$, the central $n=0$ photo-band re-appears and the original strong asymmetry of the current curve is restored.

\section{CONCLUSION AND OUTLOOK}

Our results suggest that the combination of $\mathrm{AC}$ fields and dissipation in double quantum dots leads to a rich variety of non-trivial effects. In particular, we have shown that a time-dependent monochromatic field drastically modifies the dissipative inelastic stationary current, in particular for stronger AC driving in the dynamical localisation regime. Corrections to the Tien-Gordon formula appear at larger tunnel coupling between the dots and become extremely important near zero bias in the DL regime, also in the non dissipative case.

The method presented in this work has the benefit of accounting for an arbitrary dissipative environment via the correlation function $\hat{C}_{\varepsilon}$. In the generic case, explicit analytical forms for this function are difficult to obtain and it might be easier to integrate the original equations of motion directly. Alternatively, one can numerically evaluate $\hat{C}_{\varepsilon}$ and use it as an input into our Floquet-like formalism. We also mention that the entire approach is based on the decoupling of the bosonic degrees of freedom in the polaron transformed master equation. One is therefore always restricted to the range of validity of the NIBA (non-interacting blip approximation) of the origi- 
nal spin-boson problem ${ }^{74,82}$. Discussing larger temperatures $T$ should thus lead to more reliable results as compared to the 'test-models' $C_{\varepsilon}$ which were discussed here for $T=0$.

A future extension of our approach should therefore be the derivation of a systematic perturbation theory in the electron-boson coupling, starting from the bondingantibonding basis of the double dots. In a calculation for an undriven double quantum dot, such an approach has been successfully used recently to extract dephasing and relaxation times from the frequency dependent noise spectrum 9 .

Even for the coherent case $\alpha=0$, our results have shown that there are non-trivial effects due to the combined quantum coherence inherent in the double dot, and the coherence induced by the external driving field. In particular, we found systematic corrections to standard approximation such as the Tien-Gordon formula or the rotating wave approximation. The constituing quantities $K_{m}$ and $G_{m}$ of our theory, cf. Eq. (2731), describe dissipative tunneling $\propto T_{c}^{2}$ of one additional quasi-particle between the two dots under the influence of the AC field, which again indicates that our approach is essentially perturbative in $T_{c}$, although to infinite order and exact for $\alpha=0$. We showed that partial re-summations beyond the Tien-Gordon result are justified in a non-adiabatic, high-frequency approximation, but for the general case one has to rely on a systematic evaluation of Eq. (27).

This work was supported by DFG BR 1528, EPSRC GR/R44690, the UK Quantum Circuits Network, the Nuffield foundation (T.B.), by the MCYT of Spain through the "Ramón y Cajal" program (R.A.) and project MAT2002-02465 (G.P. and R.A.). We acknowledge as well The EU Human Potential Programme under contract HPRN-CT-2000-00144.

\section{APPENDIX A: DOT OCCUPANCIES IN LAPLACE SPACE}

Here, we derive Eq. (13) for the occupancies $\left\langle n_{L / R}\right\rangle$. We define

$$
q(t) \equiv\langle p\rangle_{t} e^{-i \int_{0}^{t} d s \tilde{\varepsilon}(s)}, \quad q^{\dagger}(t) \equiv\left\langle p^{\dagger}\right\rangle_{t} e^{+i \int_{0}^{t} d s \tilde{\varepsilon}(s)}
$$

This is inserted into the equations of motion in the time domain, Eq. (8), which upon Laplace transformation become

$$
\begin{aligned}
z \hat{n}_{L}(z)-\left\langle n_{L}\right\rangle_{0} & =-i \int_{0}^{\infty} d t e^{-z t}\left\{T_{c}(t) q(t)-T_{c}^{*}(t) q^{\dagger}(t)\right\}+\Gamma_{L}\left[\frac{1}{z}-\hat{n}_{L}(z)-\hat{n}_{R}(z)\right] \\
z \hat{n}_{R}(z)-\left\langle n_{R}\right\rangle_{0} & =i \int_{0}^{\infty} d t e^{-z t}\left\{T_{c}(t) q(t)-T_{c}^{*}(t) q^{\dagger}(t)\right\}-\Gamma_{R} \hat{n}_{R}(z) \\
\hat{q}(z) & =-\frac{\Gamma_{0}}{2} \hat{q}(z) \hat{C}_{\varepsilon}(z)-i\left[\int_{0}^{\infty} d t^{\prime} e^{-z t^{\prime}} T_{c}^{*}\left(t^{\prime}\right)\left[\left\langle n_{L}\right\rangle_{t^{\prime}} \hat{C}_{\varepsilon}(z)-\left\langle n_{R}\right\rangle_{t^{\prime}} \hat{C}_{-\varepsilon}^{*}(z)\right]\right] \\
\hat{q}^{\dagger}(z) & =-\frac{\Gamma_{0}}{2} \hat{q}^{\dagger}(z) \hat{C}_{\varepsilon}^{*}(z)+i\left[\int_{0}^{\infty} d t^{\prime} e^{-z t^{\prime}} T_{c}\left(t^{\prime}\right)\left[\left\langle n_{L}\right\rangle_{t^{\prime}} \hat{C}_{\varepsilon}^{*}(z)-\left\langle n_{R}\right\rangle_{t^{\prime}} \hat{C}_{-\varepsilon}(z)\right]\right]
\end{aligned}
$$

where we used the convolution theorem in the equations for $\hat{q}(z)$ and $\hat{q}^{\dagger}(z)$ and the definitions Eq. (11). Using the definitions for the propagators $D$ and $E$, Eq. (12) we obtain upon solving for $\hat{q}^{(\dagger)}(z)$ and Laplace backtransforming,

$$
\begin{aligned}
q(t) & =-i \int_{0}^{t} d t^{\prime} T_{c}^{*}\left(t^{\prime}\right)\left[\left\langle n_{L}\right\rangle_{t^{\prime}} D_{\varepsilon}\left(t-t^{\prime}\right)-\left\langle n_{R}\right\rangle_{t^{\prime}} E_{\varepsilon}\left(t-t^{\prime}\right)\right] \\
q^{\dagger}(t) & =i \int_{0}^{t} d t^{\prime} T_{c}\left(t^{\prime}\right)\left[\left\langle n_{L}\right\rangle_{t^{\prime}} D_{\varepsilon}^{*}\left(t-t^{\prime}\right)-\left\langle n_{R}\right\rangle_{t^{\prime}} E_{\varepsilon}^{*}\left(t-t^{\prime}\right)\right],
\end{aligned}
$$

involving the propagators in the time-domain. Insertion into Eq. A2 yields 


$$
\begin{aligned}
z \hat{n}_{L}(z)-\left\langle n_{L}\right\rangle_{0} & =-\int_{0}^{\infty} d t e^{-z t} \int_{0}^{t} d t^{\prime}\left\langle n_{L}\right\rangle_{t^{\prime}}\left[T_{c}(t) T_{c}^{*}\left(t^{\prime}\right) D_{\varepsilon}\left(t-t^{\prime}\right)+T_{c}^{*}(t) T_{c}\left(t^{\prime}\right) D_{\varepsilon}^{*}\left(t-t^{\prime}\right)\right] \\
& +\int_{0}^{\infty} d t e^{-z t} \int_{0}^{t} d t^{\prime}\left\langle n_{R}\right\rangle_{t^{\prime}}\left[T_{c}(t) T_{c}^{*}\left(t^{\prime}\right) E_{\varepsilon}\left(t-t^{\prime}\right)+T_{c}^{*}(t) T_{c}\left(t^{\prime}\right) E_{\varepsilon}^{*}\left(t-t^{\prime}\right)\right]+\Gamma_{L}\left(\frac{1}{z}-\hat{n}_{L}(z)-\hat{n}_{R}(z)\right)
\end{aligned}
$$

At this point, it is useful to use a relation for a generalized convolution of a function $K\left(t, t^{\prime}\right)$ and $f\left(t^{\prime}\right)$,

$$
\begin{aligned}
& \int_{0}^{\infty} d t e^{-z t} \int_{0}^{t} d t^{\prime} K\left(t, t^{\prime}\right) f\left(t^{\prime}\right)= \\
= & \int_{0}^{\infty} d t e^{-z t} f(t) \int_{0}^{\infty} d t^{\prime} e^{-z t^{\prime}} K\left(t+t^{\prime}, t\right)
\end{aligned}
$$

which can be easily proven by substitutions. Note that the usual Laplace convolution theorem is recovered from Eq. A5 if $K\left(t, t^{\prime}\right)=K\left(t-t^{\prime}\right)$ is only a function of the difference of its two arguments. Eq. (A4) and a similar equation for $\hat{n}_{R}(z)$ then lead to Eq. (13).

\section{APPENDIX B: CALCULATION OF THE BOSON CORRELATION FUNCTION}

Explicit expressions for the bosonic correlation functions $\hat{C}_{\varepsilon}$, Eqs. (11) and Eqs. (29), which can be obtained in the zero temperature $(T=0)$ case for Ohmic dissipation. In this case,

$$
\begin{aligned}
& J(\omega)=2 \alpha \omega \exp \left(-\omega / \omega_{c}\right) \\
& C(t)=\left(1+i \omega_{c} t\right)^{-2 \alpha}, \quad g \equiv 2 \alpha .
\end{aligned}
$$

We have

$$
\begin{aligned}
\hat{C}(z) & \equiv \int_{0}^{\infty} d t e^{-z t}\left(1+i \omega_{c} t\right)^{-2 \alpha} \\
& =\left(i \omega_{c}\right)^{-2 \alpha} z^{2 \alpha-1} e^{-i z / \omega_{c}} \Gamma\left(1-2 \alpha,-i z / \omega_{c}\right),
\end{aligned}
$$

where we used Gradstein-Ryshik 3.382.4 and $\Gamma$ denotes the incomplete Gamma function. We set $\omega_{c}=1$ for a moment to simplify notations and obtain

$$
\hat{C}(-i \varepsilon)=-i(-\varepsilon)^{2 \alpha-1} e^{-\varepsilon} \Gamma(1-2 \alpha,-\varepsilon)
$$

Note that $\varepsilon$ must have a small positive imaginary part here $(\operatorname{Re} z>0$ in the definition of the Laplace transformation): the incomplete Gamma function $\Gamma(1-2 \alpha, z)$ has a branch point at $z=0$. However, we can use the series expansion

$$
\begin{aligned}
\Gamma(1-2 \alpha, x) & =\Gamma(1-2 \alpha)-\sum_{n=0}^{\infty} \frac{(-1)^{n} x^{1-2 \alpha+n}}{n !(1-2 \alpha+n)} \\
1-2 \alpha & \neq 0,-1,-2, \ldots
\end{aligned}
$$

to obtain

$$
\begin{aligned}
\hat{C}(-i \varepsilon) & =-i(-\varepsilon)^{2 \alpha-1} e^{-\varepsilon} \Gamma(1-2 \alpha) \\
& +i e^{-\varepsilon} \sum_{n=0}^{\infty} \frac{\varepsilon^{n}}{n !(1-2 \alpha+n)}, \quad 2 \alpha \neq 1,2,3, \ldots
\end{aligned}
$$

The second term is an analytic function of $\varepsilon$.

Now write

$$
\begin{aligned}
-i(-\varepsilon)^{2 \alpha-1} & =\left\{\begin{array}{cl}
-i|\varepsilon|^{2 \alpha-1}, & \varepsilon<0 \\
\varepsilon^{2 \alpha-1} e^{-\pi i(1 / 2+2 \alpha-1)} & \varepsilon>0 .
\end{array}\right. \\
& =\left\{\begin{array}{cc}
-i|\varepsilon|^{2 \alpha-1}, & \varepsilon<0 \\
\varepsilon^{2 \alpha-1}(\sin 2 \pi \alpha+i \cos 2 \pi \alpha) & \varepsilon>0
\end{array}\right.
\end{aligned}
$$

Recall the reflection formula for the Gamma function,

$$
\Gamma(1-z)=\frac{\pi}{\Gamma(z) \sin \pi z}
$$

This yields

$$
\begin{aligned}
\varepsilon>0: \quad \hat{C}(-i \varepsilon) & =\frac{\pi}{\Gamma(2 \alpha)} \varepsilon^{2 \alpha-1} e^{-\varepsilon} \\
& +i\left[\frac{\pi}{\Gamma(2 \alpha)} \varepsilon^{2 \alpha-1} e^{-\varepsilon} \cot 2 \pi \alpha\right. \\
& \left.+e^{-\varepsilon} \sum_{n=0}^{\infty} \frac{\varepsilon^{n}}{n !(1-2 \alpha+n)}\right] . \\
\varepsilon<0: \quad \hat{C}(-i \varepsilon) & =i e^{-\varepsilon}\left[-\frac{\pi}{\Gamma(2 \alpha) \sin 2 \pi \alpha}|\varepsilon|^{2 \alpha-1}\right. \\
& \left.+\sum_{n=0}^{\infty} \frac{\varepsilon^{n}}{n !(1-2 \alpha+n)}\right] .
\end{aligned}
$$

From this, we can read off the real and the imaginary part of $\hat{C}(-i \varepsilon)$. The real part is

$$
\operatorname{Re} \hat{C}(-i \varepsilon) \equiv \pi P(\varepsilon)=\frac{\pi}{\Gamma(2 \alpha)} \varepsilon^{2 \alpha-1} e^{-\varepsilon} \theta(\varepsilon)
$$

The imaginary part is

$$
\begin{aligned}
& \operatorname{Im} \hat{C}(-i \varepsilon) \equiv e^{-\varepsilon}\left[\sum_{n=0}^{\infty} \frac{\varepsilon^{n}}{n !(1-2 \alpha+n)}\right. \\
& \left.+\frac{\pi|\varepsilon|^{2 \alpha-1}}{\Gamma(2 \alpha) \sin 2 \pi \alpha} \cdot\left\{\begin{array}{ll}
-1, & \varepsilon<0 \\
\cos 2 \pi \alpha, & \varepsilon>0
\end{array}\right\}\right] \text {. }
\end{aligned}
$$


1 N. C. van der Vaart, S. F. Godijn, Y. V. Nazarov, C. J. P. M. Harmans, J. E. Mooij, L. W. Molenkamp, and C. T. Foxon, Phys. Rev. Lett. 74, 4702 (1995).

2 R. H. Blick, R. J. Haug, J. Weis, D. Pfannkuche, K. v. Klitzing, and K. Eberl, Phys. Rev. B 53, 7899 (1996).

3 T. Fujisawa, T. H. Oosterkamp, W. G. van der Wiel, B. W. Broer, R. Aguado, S. Tarucha, and L. P. Kouwenhoven, Science 282, 932 (1998).

4 R. H. Blick, D. Pfannkuche, R. J. Haug, K. v. Klitzing, and K. Eberl, Phys. Rev. Lett. 80, 4032 (1998).

5 S. Tarucha, T. Fujisawa, K. Ono, D. G. Austin, T. H. Oosterkamp, and W. G. van der Wiel, Microelectr. Engineer. 47, 101 (1999).

6 D. Loss, D. P. DiVincenzo, Phys. Rev. A 57, 120 (1998).

7 G. Burkard, D. Loss, and D. P. DiVincenzo, Phys. Rev. B 59, 2070 (1999).

8 R. H. Blick and H. Lorenz, Proceedings IEEE International Symposium on Circuits and Systems II, 245 (2000).

9 R. Aguado, T. Brandes; cond-mat/0310578 (2003).

10 L. J. Geerligs, V.F. Anderegg, P.A.M. Holweg, J.E. Mooij, H. Pothier, D. Esteve, C. Urbina, and M.H. Devoret, Phys. Rev. Lett. 64, 2691 (1990).

11 L. P. Kouwenhoven, A. T. Johnson, N. C. van der Vaart, C. J. P. M. Harmans, and C. T. Foxon, Phys. Rev. Lett. 67, 1626 (1991).

12 H. Pothier, P. Lafarge, C. Urbina, D. Esteve, and M.H. Devoret, Europhys. Lett. 17, 249 (1992).

13 (Ed.) H. Grabert and M. H. Devoret, Single Charge Tunneling, vol. 294 of NATO ASI Series B (Plenum Press, New York, 1991).

14 M. Switkes, C. M. Marcus, K. Campman, and A. C. Gossard, Science 283, 1905 (1999).

15 P. W. Brouwer, Phys. Rev. B 58, R10135 (1998).

16 M. L. Polianski and P. W. Brouwer, Phys. Rev. B 64, 075304 (2001).

17 J. N. H. J. Cremers and P. W. Brouwer, Phys. Rev. B 65, 115333 (2002).

18 M. Moskalets and M. Büttiker, Phys. Rev. B 64, 201305 (2001).

19 F. Renzoni and T. Brandes, Phys. Rev. B 64, 245301 (2001).

20 N. H. Bonadeo, J. Erland, D. Gammon, D. Park, D. S. Katzer, and D. G. Steel, Science 282, 1473 (1998).

21 T. Brandes, F. Renzoni, and R. H. Blick, Phys. Rev. B 64, 035319 (2001).

22 D. V. Averin, Solid State Comm. 105, 659 (1998).

23 D. V. Averin, in Quantum computing and quantum communications (Springer, Berlin, 1999), vol. 1509 of Lecture Notes in Computer Science, p. 413.

24 Y. Nakamura, Yu. A. Pashkin, and J. S. Tsai, Nature 398, 786 (1999).

25 J. Schliemann, D. Loss, and A. H. MacDonald, Phys. Rev. B 63, 085311 (2001).

26 Yu. Makhlin, G. Schön, and A. Shnirman, Rev. Mod. Phys. 73, 357 (2001).

27 H. A. Engel and D. Loss, Phys. Rev. Lett. 86, 4648 (2001).

28 M. Thorwart and P. Hänggi, Phys. Rev. A 65, 012309 (2002).

29 C. Bruder and H. Schoeller, Phys. Rev. Lett. 72, 1076 (1994).

${ }^{30}$ M. H. Hettler and H. Schoeller, Phys. Rev. Lett. 74, 4907
(1995)

31 C. A. Stafford and N. S. Wingreen, Phys. Rev. Lett. 76, 1916 (1996).

32 Ph. Brune, C. Bruder, and H. Schoeller, Phys. Rev. B 56, 4730 (1997).

33 T. H. Stoof and Yu. V. Nazarov, Phys. Rev. B 53, 1050 (1996).

34 Q. F. Sun, J. Wang, and T. H. Lin, Phys. Rev. B 61, 12643 (2000).

35 L. P. Kouwenhoven, S. Jauhar, K. McCormick, D. Dixon, P. L. McEuen, Yu. V. Nazarov, N. C. van der Vaart, and C. T. Foxon, Phys. Rev. B 50, 2019 (1994).

36 T. H. Oosterkamp, T. Fujisawa, W. G. van der Wiel, K. Ishibashi, R. V. Hijman, S. Tarucha, and L. P. Kouwenhoven, Nature 395, 873 (1998).

37 R. H. Blick, D. W. van der Weide, R. J. Haug, and K. Eberl, Phys. Rev. Lett. 81, 689 (1998).

38 A. W. Holleitner, H. Qin, F. Simmel, B. Irmer, R. H. Blick, J. P. Kotthaus, A. V. Ustinov, and K. Eberl, New Journal of Physics 2, 2.1 (2000).

39 H. Qin, F. Simmel, R. H. Blick, J. P. Kotthaus, W. Wegscheider, and M. Bichler, Phys. Rev. B 63, 035320 (2001).

40 H. Qin, A. W. Holleitner, K. Eberl, and R. H. Blick, Phys. Rev. B 64, 241302(R) (2001).

41 J. H. Shirley, Phys. Rev. 138, B979 (1965).

${ }^{42}$ W. Henneberger, Phys. Rev. Lett. 21, 839 (1968).

43 B. Kramer and J. Mašek, Z. Phys. B 76, 457 (1989).

44 T. Brandes, D. Weinmann, and B. Kramer, Europhys. Lett. 22, 51 (1993).

45 Y. Fu and S. C. Dudley, Phys. Rev. Lett. 70, 65 (1993).

46 M. Büttiker, A. Prêtre, and H. Thomas, Phys. Rev. Lett. 70, 4114 (1993).

47 M. Holthaus and D. W. Hone, Philosophical Magazine B 74, 105 (1996).

48 F. A. Maaø and L. Y. Gorelik, Phys. Rev. B 53, 15885 (1996).

49 M. Büttiker, J. Low Temp. Phys. 118, 519 (2000).

50 C. Zhang, Phys. Rev. B 66, 081105 (2002).

51 P. K. Tien and J. R. Gordon, Phys. Rev. 129, 647 (1963).

52 F. Grossmann, T. Dittrich, P. Jung, and P. Hänggi, Phys. Rev. Lett. 67, 516 (1991)

53 P. Johansson and G. Wendin, Phys. Rev. B 46, 1451 (1992).

54 J. Iñarrea, G. Platero, and C. Tejedor, Phys. Rev. B 50, 4581 (1994).

55 A. P. Jauho, N. S. Wingreen, and Y. Meir, Phys. Rev. B 50, 5528 (1994).

56 A. A. Ignatov, E. Schomburg, J. Grenzer, K. F. Renk, and E. P. Dodin, Z. Phys. B 98, 187 (1995).

57 M. Wagner, Phys. Rev. A 51, 798 (1995).

58 R. Aguado, J. Iñarrea, and G. Platero, Phys. Rev. B 53, 10030 (1996).

59 M. Wagner, Phys. Rev. Lett. 76, 4010 (1996).

60 M. Wagner and W. Zwerger, Phys. Rev. B 55, 10217R (1997).

61 M. Wagner and F. Sols, Phys. Rev. Lett. 83, 4377 (1999).

62 J. Lehmann, S. Kohler, P. Hänggi, and A. Nitzan, Phys. Rev. Lett. 88, 228305 (2002).

63 S. Camalet, J. Lehmann, S. Kohler, and P. Hänggi, Phys. Rev. Lett. 90, 210602 (2003). 
64 M. Sassetti and B. Kramer, Phys. Rev. B 54, R5203 (1996).

${ }^{65}$ G. Cuniberti, M. Sassetti, and B. Kramer, Phys. Rev. B 57, 1515 (1998).

66 A. Fechner, M. Sassetti, B. Kramer, and E. Galleani d'Agliano, Phys. Rev. B 64, 195315 (2001).

67 K.-V. Pham, Eur. Phys. J. B 36, 607 (2003).

${ }^{68}$ M. Vicar, A. Braggio, E. Galleani d'Agliano, and M. Sassetti, Eur. Phys. J. B 25, 115 (2002).

69 R. López, R. Aguado, G. Platero, and C. Tejedor, Phys. Rev. Lett. 81, 4688 (1998).

70 A. Kaminski, Yu. V. Nazarov, and L. I. Glazman, Phys. Rev. Lett. 83, 384 (1999).

71 R. Aguado and G. Platero, Phys. Rev. Lett. 81, 4971 (1998).

72 C. E. Creffield and G. Platero, Phys. Rev. B 65, 113304 (2002).

73 C. E. Creffield and G. Platero, Phys. Rev. B 66, 235303 (2002).

74 M. Grifoni and P. Hänggi, Phys. Rep. 304, 229 (1998).

75 S. A. Gurvitz and Ya. S. Prager, Phys. Rev. B 53, 15932 (1996).
76 S. A. Gurvitz, Phys. Rev. B 57, 6602 (1998).

77 A. J. Leggett, S. Chakravarty, A. T. Dorsey, M. P. A. Fisher, A. Garg, and W. Zwerger, Review of Modern Physics 59, 1 (1987).

78 T. Brandes and T. Vorrath, Phys. Rev. B 66, 075341 (2002).

79 T. Hayashi, T. Fujisawa, H.-D. Cheong, Y.-H. Jeong, Y. Hirayama, Phys. Rev. Lett. 91, 226804 (2003).

80 T. Brandes and B. Kramer, Phys. Rev. Lett. 83, 3021 (1999).

81 T. Brandes, T. Vorrath, Int. J. Mod. Phys. B 28, 5465 (2003).

82 U. Weiss, Quantum Dissipative Systems, vol. 2 of Series of Modern Condensed Matter Physics (World Scientific, Singapore, 1993).

83 J. C. A. Barata and W. F. Wreszinski, Phys. Rev. Lett. 84, 2112 (2000).

${ }^{84}$ L. Allen and J. H. Eberly, Optical Resonance and TwoLevel Atoms (Dover, New York, 1987).

85 For a recent review see, e.g., G. Platero, R. Aguado; cond-mat/0311001 (2003). 